\title{
A study of the effect of ionization and illumination on morphologies of planetary nebulae
}

\author{
Yong Zhang ${ }^{1}$, Sun Kwok ${ }^{1}$ and Chung-Ming Ko ${ }^{1,2}$ \\ ${ }^{1}$ Department of Physics, The University of Hong Kong, Hong Kong, China \\ email: zhangy96@hku.hk \\ ${ }^{2}$ Institute of Astronomy, Department of Physics and Center for Complex Systems, National \\ Central University, Taiwan
}

\begin{abstract}
We carry out a modelling study of the effect of ionization and illumination on the morphologies of planetary nebulae (PNs), aiming to investigate the hypothesis of Kwok (2010) that the bipolar and multipolar lobes of PNs can be produced by leakage of UV photons into those directions. Using photoinization code, we construct a series of nebular models consisting of a dense envelope and cones of low density. The results show that the visible morphologies of PNs may be fundamentally different from their intrinsic structures.
\end{abstract}

Keywords. planetary nebulae: general, circumstellar matter

With increased dynamic range of space observations, a number of PNs have been found to exhibit multipolar structures. The physical mechanisms responsible for generating such structures are not understood and a variety of hydrodynamical models have been constructed to simulate such structures (see the reference in Balick \& Frank 2002). Kwok (2010) presented an alternative scenario that the visible nebulosity in fact represents lowdensity regions cleared by fast outflows, and the similar lobe sizes of multipolar PNs are not due to simultaneous ejection of matter in different directions, but by leakage of UV photons in these directions. Here we present a preliminary investigation of this possibility using photoionization model.

The models were made using the photoionization code CLOUDY (Ferland et al. 1998). We assume that the nebula consists of two components: a spherical envelope with a hydrogen density of $10^{5} \mathrm{~cm}^{-3}$ and a pair of symmetrical cones with a hydrogen density of $10^{3} \mathrm{~cm}^{-2}$ and an open angle of 45 degrees (Fig. 1). The spherical envelope represents the circumstellar matter ejected by AGB winds, and the cones are the regions cleared by fast outflows. The model results clearly show that the visible $\mathrm{H} \beta$ appearance of a a young PN having a low-temperature central star cannot represent its real structure (Fig. 1). This is because the extended high-density envelope is optical thick to the UV photons (ionization bounded), while the low-density cones are fully photonionized. The bright region in fact represents regions of lower density.

Since the temperatures of the central stars increase with the nebular evolution, we have also investigated the effect of evolution by making models with different central temperatures. As shown in Fig. 1, when the temperature of the central star is low, the PN shows bipolar structure, and with the evolution the apparent structure becomes spherical. This exercise suggests that it is not necessary to introduce hydrodynamic mechanism to account for the change of nebular appearances.

The observed nebular appearances could also be misleading due to various observation biases. In Fig. 2, we show the visible appearances of our modelled PN under different orientation angles and instrumental sensitivities. It is clear that pole-on PNs can be more 

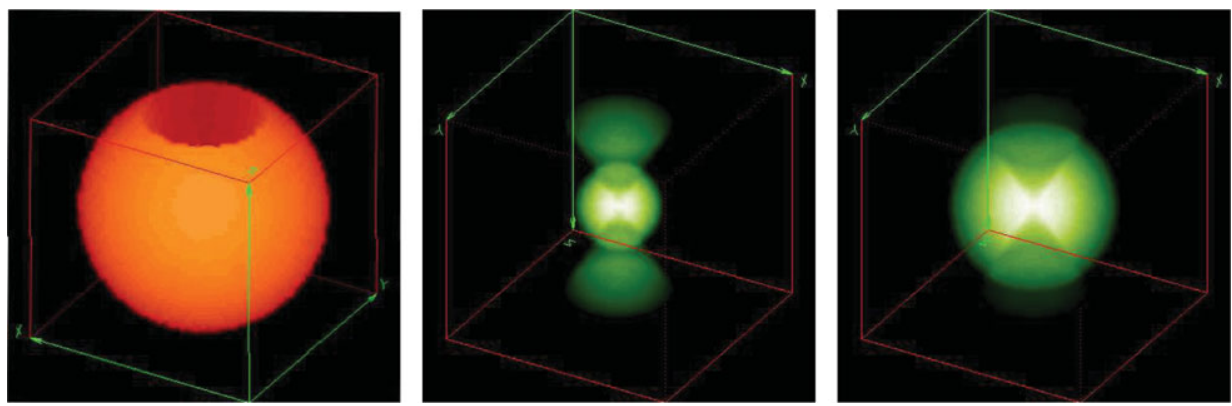

Figure 1. The assumed nebular structure for the modelling (left panel), and The calculated $\mathrm{H} \beta$ appearance of a young PN (middle panel) and a more evolved PN (right panel).

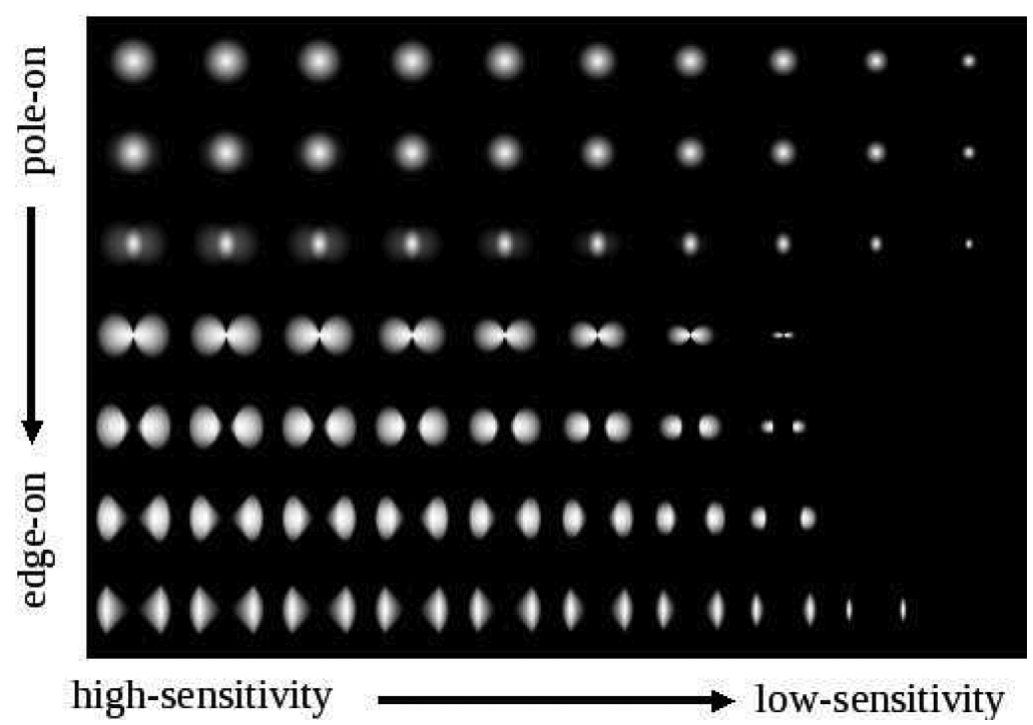

Figure 2. Nebular appearances under different orientation angles and instrumental sensitivities. From up to down, the orientation angles are $0,15,30,45,60,75$, and 90 degree, respectively.

easily detected because of higher emission measures. When the sensitivity is low, PNs appear as round or elliptical appearance.

In summary, we verify the hypothesis that illumination of UV photons can significantly affect the appearances of PNs. The classification of nebular morphologies can also be influenced by observational biases. Deep multi-wavelength observations are essential to reveal nebular intrinsic structures.

\section{Acknowledgements}

The work was supported by a grant from the Research Grants Council of the Hong Kong Special Administrative Region, China (Project No. HKU 7031/10P) and a grant to YZ from the Seed Funding Programme for Basic Research in HKU (200909159007). CMK is supported in part by the National Science Council, Taiwan, under grants NSC 98-2923-M-008-001-MY3 and NSC 99-2112-M-008-015-MY3.

\section{References}

Balick, B. \& Frank, A. 2002, ARA\&A, 40, 439

Ferland, G. J., et al. 1998, PASP, 110, 761

Kwok, S. 2010, PASA, 27, 174 